\title{
InGaAs and GaAs Quantum Dot Solar Cells Grown by Droplet Epitaxy
}

Peng $\mathrm{Yu}^{a}$, Jiang $W u^{b^{*}}$, Lei Gao ${ }^{c}$, Huiyun Liu ${ }^{b}$, Zhiming Wang ${ }^{a^{*}}$

anstitute of Fundamental and Frontier Science, University of Electronic Science and Technology of China, Chengdu 610054, P.R. China

${ }^{b}$ Department of Electronic and Electrical Engineering, University College London, Torrington Place, London WC1E 7JE, United Kingdom

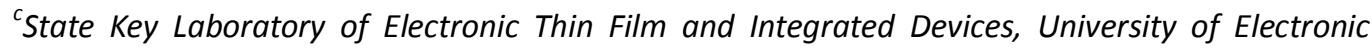
Science and Technology of China, Chengdu 610054, P.R. China

\begin{abstract}
Traditional p-i-n junction solar cells imbedded with quantum dots are attractive to achieve chromatic light absorption enhancement. In this paper, multi-layer stacked GaAs and $\operatorname{In}_{0.1} \mathrm{Ga}_{0.9} \mathrm{As}$ quantum dots grown by the droplet epitaxy technique are sandwiched between $\mathrm{Al}_{0.4} \mathrm{Ga}_{0.6} \mathrm{As}_{\text {layers }}$ for solar energy harvesting. The performance of GaAs and InGaAs quantum dot solar cells is compared using structural, optical, and electrical measurements. Two-step photon absorption process is studied via adding external infrared pumping sources in quantum efficiency measurements at room temperature. This work demonstrates that strain-free nanostructures by droplet epitaxy are promising for photovoltaic application.
\end{abstract}

Key words: Quantum dots; solar cells; drop epitaxy; intraband; interband; intermediate band. 


\section{Introduction}

In order to boost the efficiency of p-n junction solar cells to exceed the Shockley-Queisser limit, the concept of quantum dot solar cells (QDSCs) has been proposed to achieve high efficiency solar cells. It was predicted that QDSCs have the potential to implement the intermediate band concept and increase efficiencies to $\sim 45 \%$ under one sun and $63 \%$ under full concentration in a single p-n junction [1-3]. Quantum dots (QDs) incorporated in solar cells can give rise to multiple exciton generation [4], hot carrier transfer [1], and multiple transitions [5]. Particularly, the QD-based intermediate band solar cells (IBSCs) allow multiple transitions via two-step sub-bandgap absorption because of the discrete density of states of QDs. Martí et al. reported photocurrent production from two-step optical transitions via IB albeit under a cryogenic temperature [6]. Okada et al. showed direct Si-doping InAs QDSCs with a clear photocurrent production at room temperature owing to two-step absorption of sub-bandgap photons [7, 8]. III-V materials, such as GaAs, InAs, and InGaAs QDs are commonly inserted in a p-n junction of the barrier materials to obtain IBSCs [9-12]. Although the InAs/GaAs QD structure is widely studied, the performance of InAs/GaAs systems is impaired by the buildup of the internal strain during stacking QD layers (7\% lattice mismatch). $\mathrm{In}_{\mathrm{x}} \mathrm{Ga}_{1-\mathrm{x}} \mathrm{As}$ and GaAs QDs are alternative candidates for avoiding the large lattice mismatch with GaAs substrates. Sugaya et al. achieved 400-layer $\mathrm{In}_{0.4} \mathrm{Ga}_{0.6} \mathrm{As}$ QDs without introducing any strain balancing technique [12]. As a result, no obvious degradation in crystal quality was observed even after stacking 400 QD layers. The mainstream growth method for self-organization QDs is the Stranski-Krastanow (S-K) growth mode based on lattice-mismatched materials using molecular beam epitaxy (MBE) and/or metal organic chemical vapor deposition (MOCVD). The lattice mismatch results in accumulation of strain and thus generation of defects, restricting the number of stacked layers. Although the insertion of strain-compensation layers between QDs can alleviate the strain accumulation, the strain balance technique requires precise control in growth and imposes constrains on design flexibility. So far, researches on QDSCs are focusing on enhancing optical absorption in QDs, including optimization of the QD growth, improving solar cell structures, and engineering light trapping techniques $[13,14]$. Among these efforts, droplet epitaxy (DE) growth technique shows its advantages on the fabrication of strain-free QDs $[15,16]$. It was reported that DE allows the selforganization of strain-free GaAs QDs in AlGaAs barriers [17]. Two-photon absorption in strain-free GaAs/AlGaAs QDSCs grown via the DE method was confirmed [18]. While there are still opening questions to address to make QDSCs more efficient solar cells, such as the open-circuit voltage $\left(\mathrm{V}_{\mathrm{oc}}\right)$ degradation and marginal photocurrent contribution from QDs, solar cells based on strain-free QDs are expected to improve cell performance because of the alleviation of strain-induced defects. Strain-free ring-shaped QDSCs were reported with an improved $V_{\text {oc }}$ by post-growth thermal annealing [10]. Without the insertion of compensating layers, the number of DE QD layers can be much higher than that of the S-K QDs. Additionally, an areal density of 7.3 $\times 10^{11} \mathrm{~cm}^{2}$ GaAs QDs was achieved at a growth temperature of $30^{\circ} \mathrm{C}$ by controlling the amount of Ga [19]. Compared with S-K method, locally stacked nanostructures can also be fabricated to increase absorption by droplet epitaxy [20]. InGaAs QDs without a wetting layer on GaAs (001) substrates were reported using the DE method with highly dense Ga droplets [21]. In this paper, we investigate QDSCs with the incorporation of $\mathrm{GaAs} / \mathrm{Al}_{0.4} \mathrm{Ga}_{0.6} \mathrm{As}$ QDs and 
$\mathrm{In}_{0.1} \mathrm{Ga}_{0.9} \mathrm{As} / \mathrm{Al}_{0.4} \mathrm{Ga}_{0.6} \mathrm{As}$ QDs grown via the DE method. The optical inter-subband transitions on the solar cell performance are evaluated by external quantum efficiency measurements at room temperature.

\section{Experimental Details}

Atomic force microscopy (AFM) was used to determine the sizes and densities of the GaAs and $\mathrm{In}_{0.1} \mathrm{Ga}_{0.9} \mathrm{As}$ QDs. Figure 1 (a) and (b) show the $1 \times 1 \mu \mathrm{m}^{2}$ AFM images of uncapped GaAs QDs and InGaAs QDs, which yield densities of $3.43 \times 10^{10} / \mathrm{cm}^{2}$ and $3.46 \times 10^{10} / \mathrm{cm}^{2}$ respectively, comparable to that of S-K QDs. The mean diameters of GaAs and $\mathrm{In}_{0.1} \mathrm{Ga}_{0.9}$ As QDs are $40 \mathrm{~nm}$. Figure 1 (c) and (d) illustrate the solar cell structures with 10 layers of GaAs and $\operatorname{In}_{0.1} \mathrm{Ga}_{0.9}$ As QDs sandwiched in the $\mathrm{Al}_{0.4} \mathrm{Ga}_{0.6} \mathrm{As}$ matrix. The samples were grown on $\mathrm{n}^{+}$-GaAs substrates started with a $100 \mathrm{~nm} \mathrm{n}{ }^{+}$-GaAs buffer layer, $30 \mathrm{~nm}$ AlGaAs BSF, $500 \mathrm{~nm}$-AlGaAs base and $50 \mathrm{~nm}$ AlGaAs spacer, sequentially. Ten periods of QDs were then grown via the DE method at $400{ }^{\circ} \mathrm{C}$. The difference between the two samples is only the QDs, which are made of 4 MLs of GaAs and $\mathrm{In}_{0.1} \mathrm{Ga}_{0.9} \mathrm{As}$, respectively. Each layer of QDs was separated by $50 \mathrm{~nm} \mathrm{Al}_{0.4} \mathrm{Ga}_{0.6} \mathrm{As}$ spacer, of which $10 \mathrm{~nm}$ AlGaAs was deposited at $400{ }^{\circ} \mathrm{C}$ and $40 \mathrm{~nm} \mathrm{Al} \mathrm{Al}_{0.4} \mathrm{Ga}_{0.6}$ As at $590{ }^{\circ} \mathrm{C}$. A $200 \mathrm{~nm}$ p- $\mathrm{Al}_{0.8} \mathrm{Ga}_{0.2} \mathrm{As}$ was deposited as the emitter. Finally, a $30 \mathrm{~nm}$ p- $\mathrm{Al}_{0.8} \mathrm{Ga}_{0.2} \mathrm{As}$ window was deposited to curb surface recombination, and a $50 \mathrm{~nm} \mathrm{p+-GaAs} \mathrm{layer} \mathrm{was} \mathrm{grown} \mathrm{as} \mathrm{the} \mathrm{contact}$ layer. Metal contacts were deposited on the top and rear of the solar cells by standard photolithography, thermal evaporation, and lift-off. Ti/Pt/Au (50/100/300 nm) was deposited as p-type metal contact and $\mathrm{Ni} / \mathrm{AuGe} / \mathrm{Au}(20 / 100 / 100 \mathrm{~nm})$ as n-type back contact. Ohmic contacts were formed by rapid thermal annealing at $400{ }^{\circ} \mathrm{C}$ for 30 seconds under nitrogen atmosphere. No anti-reflection coating was deposited on these devices. The photoluminescence (PL) was measured using a Nanometrics RPM2000 rapid photoluminescence mapper at room temperature. The dark current was measured from 80 to $300 \mathrm{~K}$ using an ARS wafer probe (Advanced Research System, Inc) interfaced with a Keithley 2400 source meter. The performance of the QDSCs was measured by a SAN-EI solar simulator under standard conditions of AM1.5G, $100 \mathrm{~mW} / \mathrm{cm}^{2}$, and $25^{\circ} \mathrm{C}$. The external quantum efficiency (EQE) of GaAs and $\mathrm{In}_{0.1} \mathrm{Ga}_{0.9}$ As QDSCs was characterized by a QEX10 Quantum Efficiency Measurement System (PV Measurements, Inc). To confirm the interband and intraband transitions, a continuous wave (CW) infrared (IR) laser source was illuminated on the sample to measure the change of EQE. The interband transition was pumped by monochromatic light from the monochromator of QEX10 system. The intraband transition was pumped by $905,980,1064 \mathrm{~nm}$ infrared lasers with nominal power of $40 \mathrm{~mW}$. 


\section{a}

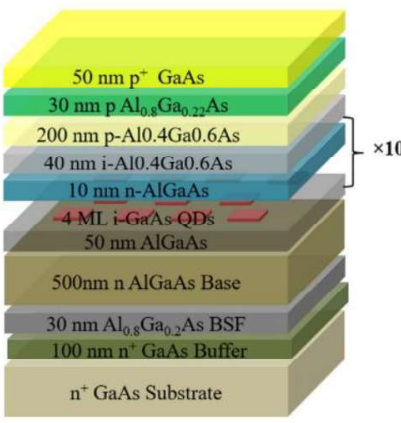

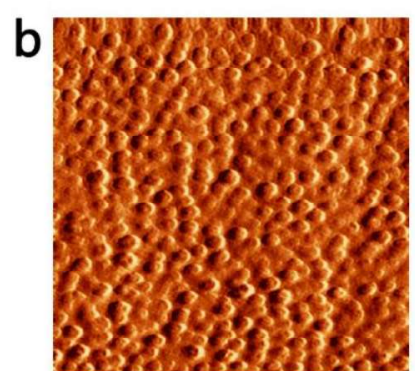

d

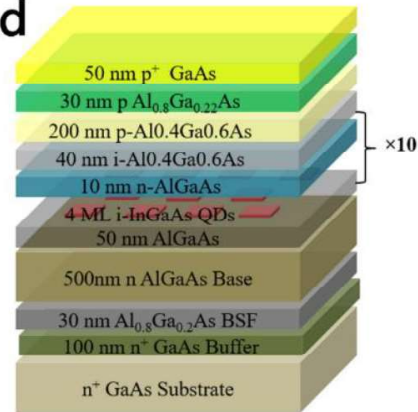

Fig. 1. (a) (b) AFM image of $1 \times 1 \mu \mathrm{m}^{2}$ GaAs QDs and $\mathrm{In}_{0.1} \mathrm{Ga}_{0.9}$ As QDs on an AlGaAs surface grown by DE, respectively. (c) (d) Schematics of the GaAs and $\operatorname{In}_{0.1} \mathrm{Ga}_{0.9}$ As QDSCs.

\section{Results and Discussion}

Figure 2 (a) is the energy band schematic of GaAs and $\operatorname{In}_{0.1} \mathrm{Ga}_{0.9} \mathrm{As}$ QDSCs. The intersubband transitions provide an extra photocurrent. The total bandgap is $1.93 \mathrm{eV}$, near the optimal value of $1.95 \mathrm{eV}$ for IBSCs [22]. The presence of the QD quantized energy levels is confirmed by PL emission spectra from the samples measured at room temperature as shown in Fig. 2 (b). The first peak $(860 \mathrm{~nm}, 1.44 \mathrm{eV})$ is attributed to the optical transition from the QD energy levels. Weak emission peaks shown at $1.25 \mathrm{eV}(991 \mathrm{~nm})$ and $1.22 \mathrm{eV}(1017 \mathrm{~nm})$ for InGaAs QDs and GaAs QDs, respectively, are due to defects in DE QDs. Various defects, such as antisites and point defects, can formed in low temperature DE and serve as non-radiative recombination centers [23]. Such defects can be reduced or eliminated by optimizing the crystallization conditions. Rapid thermal annealing is used to improve the quality of InGaAs and GaAs QDs grown at low temperature via the DE method [10, 24]. However, the emission from InGaAs QDs is much weaker than that of the GaAs QDs because of the additional strain in the InGaAs QDs. 
a

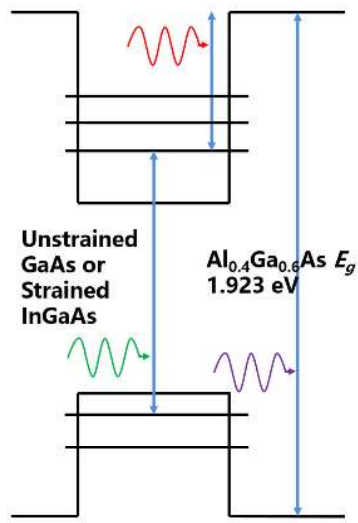

b

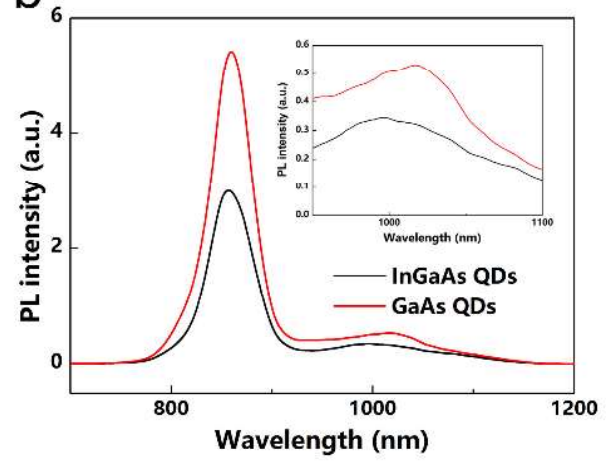

Fig. 2 (a) Schematic energy band diagram of multiple GaAs/AlGaAs and $\mathrm{In}_{0.1} \mathrm{Ga}_{0.9} \mathrm{As} / \mathrm{AlGaAs}$ QDs. (b) PL spectra of GaAs and $\mathrm{In}_{0.1} \mathrm{Ga}_{0.9}$ As QDs measured with excitation power of $\sim 400 \mathrm{~W} / \mathrm{cm}^{2}$ at $300 \mathrm{~K}$. The inset is the magnified PL peaks measured from GaAs and $\mathrm{In}_{0.1} \mathrm{Ga}_{0.9}$ As QDs.

The dark current-voltage characteristics for GaAs QDSCs and $\mathrm{In}_{0.1} \mathrm{Ga}_{0.9}$ As QDSCs are shown in Fig. 3 (a) and (b). The dark current-voltage characteristics were measured at different temperatures. As the temperature increases, the dark current increases due to the thermionic emission of the carriers. The large saturation current of both cells suggests the possible presence of defects in QDs [25]. The $\mathrm{In}_{0.1} \mathrm{Ga}_{0.9}$ As QDSCs exhibit a more complicated behavior: it has a higher current at negative bias and a lower current at positive bias than that of the GaAs QDSCs, indicating more defects in $\mathrm{In}_{0.1} \mathrm{Ga}_{0.9}$ As QDSCs cells. The short-circuit current density $\left(\mathrm{J}_{\mathrm{sc}}\right)$ curves in Fig. 3 (c) show that the both cells have low fill factors (FF), $43.1 \%$ and $34.2 \%$ for GaAs and $\operatorname{In}_{0.1} \mathrm{Ga}_{0.9} \mathrm{As}$ QDSCs, respectively. The poor FF is caused by a low shunt resistance that can be attributed to low crystalline of QDs and AlGaAs layers grown at low temperatures $[10,26]$. These defects can be reduced or eliminated by rapid thermal annealing process. The $\mathrm{V}_{\text {oc }}$ of QDSCs and $\mathrm{In}_{0.1} \mathrm{Ga}_{0.9} \mathrm{As}$ QDSCs are 0.92 and $0.60 \mathrm{~V}$, respectively. Compared with the strain-free GaAs/AlGaAs QDSCs, the $\mathrm{V}_{\text {oc }}$ drop in InGaAs/AlGaAs QDSCs is primarily due to the lattice mismatch and strain-induced defects. The strain-induced defects may lead to additional non-radiative recombination and inefficient collection of the carriers in the QD regions. Various papers have reported a distinct degradation in $\mathrm{V}_{\mathrm{oc}}$ due to carrier recombination in QDSCs [27, 28]. Figure 3 (d) plots the EQE spectra of two samples. The low quantum efficiency in 700-900 nm is mainly due to the weak absorption of the QDs. GaAs and $\mathrm{In}_{0.1} \mathrm{Ga}_{0.9}$ As QDSCs only contribute an efficiency of $0.72 \%$ and $0.11 \%$ respectively due to the low absorption volume and non-optimized growth conditions at the low temperature as discussed above. Nonetheless, the strain-free GaAs QDSC significantly outperforms the slightly strained InGaAs QDSC from both JV and EQE measurements. 


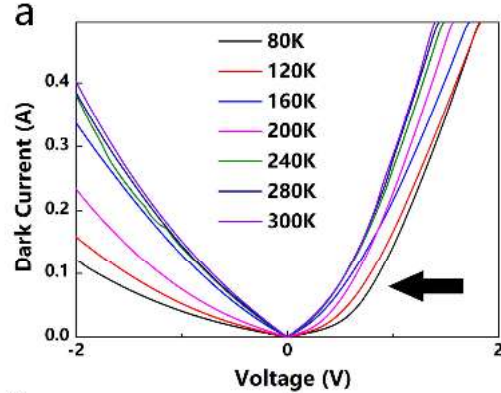

c

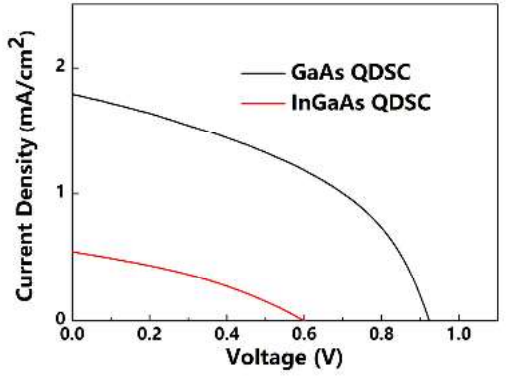

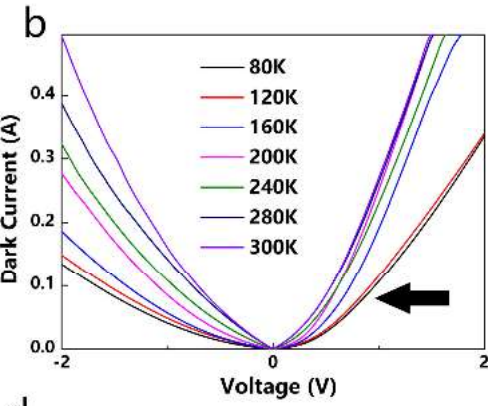

d

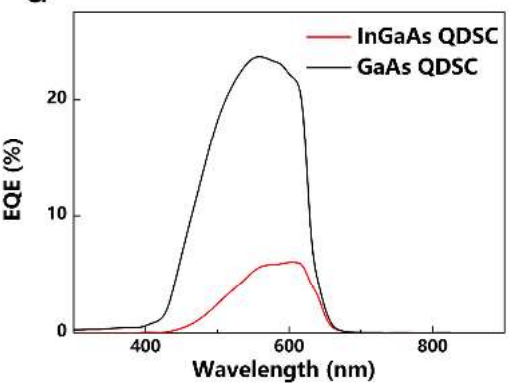

Fig. 3 The dark current-voltage characteristics of (a) GaAs QDSCs (b) $\mathrm{In}_{0.1} \mathrm{Ga}_{0.9}$ As QDSCs measured from 80 to $300 \mathrm{~K}$. (c) Current-voltage characteristics of GaAs QDSCs and $\operatorname{In}_{0.1} \mathrm{Ga}_{0.9}$ As QDSCs measured under standard AM1.5 G 1 sun illumination at room temperature. (d) External quantum efficiency of GaAs QDSCs and $\operatorname{In}_{0.1} \mathrm{Ga}_{0.9}$ As QDSCs at room temperature.

Given the weak absorption of QDs at room temperature, it is difficult to observe the contribution of QDs above $860 \mathrm{~nm}$ but possible to study the intraband and interband transitions by adding a second pumping source. The interband transitions refer to VB to IB and VB to CB, while the wavelengths of infrared lasers were chosen to be long enough so that the laser beam can only excite the intraband transitions, from IB to CB. Figure 4 (a) presents the schematic measurement setup to characterize photocurrent production as a direct result of optical transitions of electrons from intraband and interband transitions at room temperature. The energy band offsets between GaAs and InGaAs QD ground states and AlGaAs conduction band edge are less than $0.5 \mathrm{eV}$ and the ground state transitions in both QDs are about $1.44 \mathrm{eV}$. Therefore, the lasers, $1064 \mathrm{~nm}(1.16$ $\mathrm{eV}), 905 \mathrm{~nm}(1.37 \mathrm{eV})$, and $980(1.27 \mathrm{eV})$ can only excite the intraband transitions and pump the electrons from IB to CB. As shown in Fig. 4 (b) and (c), although the EQE spectra above GaAs bandgap are overwhelmed in noise, a distinct enhancement is observed from $400 \mathrm{~nm}$ to $560 \mathrm{~nm}$. The $1064 \mathrm{~nm}$ light source has led a maximum EQE enhancement while the 905 and $908 \mathrm{~nm}$ lasers demonstrate a lower enhancement. The $1064 \mathrm{~nm}$ source is able to efficiently drive the electrons on the IB to CB. However, the 905 and 908 sources have lower impact on the transition of IB-CB. Additionally, the enhancement observed in GaAs QDs is clearly higher than that measured in InGaAs QDs, which again confirms no lattice-mismatch is preferred under the same growth conditions. Negative $\triangle \mathrm{EQE}$ occurs from 570 to $660 \mathrm{~nm}$. A possible interpretation of negative $\triangle \mathrm{EQE}$ at the long wavelength region from 570 to $660 \mathrm{~nm}$ can be ascribed to the fact that no QD is presented in the AlGaAs base region, where most long-wavelength photons are absorbed, and thus no additional current is generated from the secondary excitation. On the other hand, enhanced nonradiative recombination of carriers via the trap states may be enhanced under the second IR illumination, as illustrated in Fig. 5. This is in agreement with the observation reported in ref. [3]. 
When mid-gap trap states are presented, the IR excited photocarriers from QDs are then annihilated resulting in the EQE reduction. At the surface of solar cells, there are some unoccupied antibonding states which locate within the CB continuum [3]. The additional infrared excitation can also lead to nonradiative loss of photocarriers via higher energy trap states. The negative and positive $\triangle \mathrm{EQE}$ depends on the amount of photocarriers in QDs and the conduction band. If it is low and limited, the negative $\triangle \mathrm{EQE}$ appears; if the amount of carriers is high, the pumped carriers contribute to photocurrent can compensate the annihilated carriers during recombination.
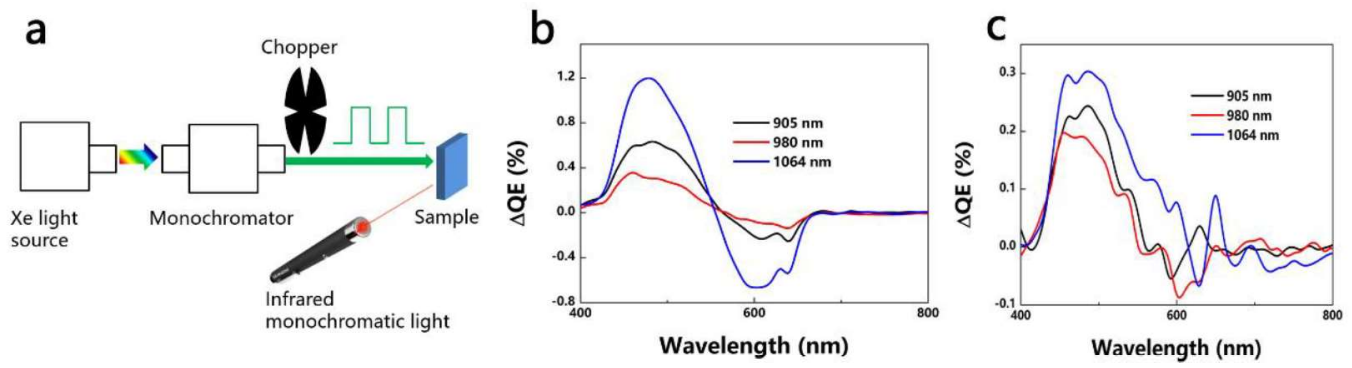

Fig. 4 (a) Schematic measurement setup to characterize $\triangle \mathrm{EQE}$ as a direct result of optical transitions of electrons from IB to $\mathrm{CB}$. The illumination of low-energy photons from this IR source can then pump the electrons only from IB to CB. (b) and (c) $\triangle E Q E$ spectra of GaAs QDSCs and $\operatorname{In}_{0.1} \mathrm{Ga}_{0.9}$ As QDSCs. Since the weak EQE spectrum of the InGaAs QDSC, the fluctuation in Fig. 4 (c) may originate from the uncertainty i.e. noise.

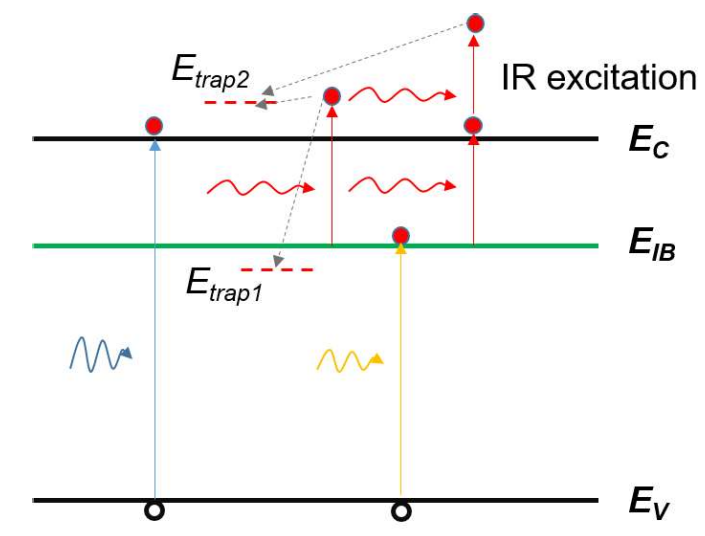

Fig. 5 Trap-assisted recombination in QD solar cells: IR excitation create additional hot carriers that recombine non-radiatively via trap states in the bandgap and conduction band.

Due to the relative low optical quality of the nanostructures and AlGaAs spacer layers obtained at low growth temperatures, the performances of these prototype devices are still far from satisfactory. In addition to QDs and quantum rings, a rich spectrum of nanostructures can be fabricated via the DE method, such as nanorods, nanoholes, dome-shaped nanocrystals, square-shaped nanocrystals, pentagon-shaped nanocrystals and coupled quantum structures [29]. These nanostructures are promising for optoelectronic applications. Till now, most work on droplet epitaxy focuses on fabrication, optical properties and understanding the growth mechanisms of nanostructures while device applications of these nanostructures are much less explored. Future efforts in solar cells based on the DE method are still desired. 


\section{Conclusion}

In conclusion, GaAs/AlGaAs and InGaAs/AlGaAs quantum dots are fabricated via droplet epitaxy (DE) method to achieve zero strain or low strain photovoltaic devices. PL measurements are performed to examine the optical properties of the QDs. Electrical measurements including EQE and $\mathrm{J}-\mathrm{V}$ characteristics were carried out, and confirm higher device performance with strain-free nanostructures. Although the QDSCs were grown under the same conditions, the GaAs QDSC has a better performance than InGaAs QDSC, because of the small lattice mismatch of GaAs/AlGaAs QDs $(0.25 \%$ verse $0.69 \%) . \mathrm{In}_{\mathrm{x}} \mathrm{Ga}_{1-\mathrm{x}} \mathrm{As}$ can be further tailored by the DE method to match the barriers to form a lattice-matched system for high efficiency IBSCs. By adding infrared sources to excite the intraband transitions in EQE measurements, an additional photocurrent from the QD layers was observed at room temperature. This study demonstrates the potential of using DE method to create QD based IBSCs using materials preferred for ideal IBSCs.

\section{Acknowledgement}

This work was supported by National Program on Key Basic Research Project (973 Program) 2013CB933301, National Natural Science Foundation of China 51302030 and National Natural Science Foundation of China 61474015.

\section{References}

[1] A. Luque, A. Marti, Increasing the efficiency of ideal solar cells by photon induced transitions at intermediate levels, Physical Review Letters, 78 (1997) 5014-5017.

[2] G. Wei, K.-T. Shiu, N.C. Giebink, S.R. Forrest, Thermodynamic limits of quantum photovoltaic cell efficiency, Applied Physics Letters, 91 (2007) 223507.

[3] R. Tamaki, Y. Shoji, Y. Okada, K. Miyano, Spectrally resolved intraband transitions on two-step photon absorption in InGaAs/GaAs quantum dot solar cell, Applied Physics Letters, 105 (2014) 073118.

[4] O.E. Semonin, J.M. Luther, S. Choi, H.Y. Chen, J.B. Gao, A.J. Nozik, M.C. Beard, Peak External Photocurrent Quantum Efficiency Exceeding 100\% via MEG in a Quantum Dot Solar Cell, Science, 334 (2011) 1530-1533.

[5] J. Wu, D.L. Shao, Z.H. Li, M.O. Manasreh, V.P. Kunets, Z.M. Wang, G.J. Salamo, Intermediate-band material based on GaAs quantum rings for solar cells, Applied Physics Letters, 95 (2009) 3.

[6] A. Martí, E. Antolín, C.R. Stanley, C.D. Farmer, N. López, P. Díaz, E. Cánovas, P.G. Linares, A. Luque, Production of Photocurrent due to Intermediate-to-Conduction-Band Transitions: A Demonstration of a Key Operating Principle of the Intermediate-Band Solar Cell, Physical Review Letters, 97 (2006) 247701. 
[7] Y. Okada, T. Morioka, K. Yoshida, R. Oshima, Y. Shoji, T. Inoue, T. Kita, Increase in photocurrent by optical transitions via intermediate quantum states in direct-doped InAs/GaNAs strain-compensated quantum dot solar cell, Journal of Applied Physics, 109 (2011) 024301.

[8] S. Yasushi, A. Katsuhiro, O. Yoshitaka, Self-organized InGaAs/GaAs quantum dot arrays for use in high-efficiency intermediate-band solar cells, Journal of Physics D: Applied Physics, 46 (2013) 024002.

[9] G. Jolley, L. Fu, H.F. Lu, H.H. Tan, C. Jagadish, The role of intersubband optical transitions on the electrical properties of InGaAs/GaAs quantum dot solar cells, Progress in Photovoltaics: Research and Applications, 21 (2013) 736-746.

[10] J. Wu, Z.M. Wang, V.G. Dorogan, S. Li, Z. Zhou, H. Li, J. Lee, E.S. Kim, Y.I. Mazur, G.J. Salamo, Strain-free ring-shaped nanostructures by droplet epitaxy for photovoltaic application, Applied Physics Letters, 101 (2012) 043904.

[11] D. Guimard, R. Morihara, D. Bordel, K. Tanabe, Y. Wakayama, M. Nishioka, Y. Arakawa, Fabrication of InAs/GaAs quantum dot solar cells with enhanced photocurrent and without degradation of open circuit voltage, Applied Physics Letters, 96 (2010) 203507.

[12] T. Sugaya, O. Numakami, R. Oshima, S. Furue, H. Komaki, T. Amano, K. Matsubara, Y. Okano, S. Niki, Ultra-high stacks of InGaAs/GaAs quantum dots for high efficiency solar cells, Energy \& Environmental Science, 5 (2012) 6233-6237.

[13] Z. Zheng, H. Ji, P. Yu, Z. Wang, Recent Progress Towards Quantum Dot Solar Cells with Enhanced Optical Absorption, Nanoscale Research Letters, 11 (2016) 1-8.

[14] J. Wu, P. Yu, A.S. Susha, K.A. Sablon, H.Y. Chen, Z.H. Zhou, H.D. Li, H.N. Ji, X.B. Niu, A.O. Govorov, A.L. Rogach, Z.M.M. Wang, Broadband efficiency enhancement in quantum dot solar cells coupled with multispiked plasmonic nanostars, Nano Energy, 13 (2015) 827-835.

[15] K. Nobuyuki, I. Keiko, Growth of GaAs Epitaxial Microcrystals on an S-Terminated GaAs Substrate by Successive Irradiation of Ga and As Molecular Beams, Japanese Journal of Applied Physics, 32 (1993) 2052.

[16] J. Wu, D.L. Shao, V.G. Dorogan, A.Z. Li, S.B. Li, E.A. DeCuir, M.O. Manasreh, Z.M. Wang, Y.I. Mazur, G.J. Salamo, Intersublevel Infrared Photodetector with Strain-Free GaAs Quantum Dot Pairs Grown by High-Temperature Droplet Epitaxy, Nano Letters, 10 (2010) 1512-1516.

[17] T. Mano, T. Kuroda, K. Mitsuishi, M. Yamagiwa, X.J. Guo, K. Furuya, K. Sakoda, N. Koguchi, Ring-shaped GaAs quantum dot laser grown by droplet epitaxy: Effects of post-growth annealing on structural and optical properties, Journal of Crystal Growth, 301-302 (2007) 740-743.

[18] A. Scaccabarozzi, S. Adorno, S. Bietti, M. Acciarri, S. Sanguinetti, Evidence of two-photon absorption in strain-free quantum dot GaAs/AIGaAs solar cells, physica status solidi (RRL) - Rapid Research Letters, 7 (2013) 173-176.

[19] M. Jo, T. Mano, Y. Sakuma, K. Sakoda, Extremely high-density GaAs quantum dots grown by droplet epitaxy, Applied Physics Letters, 100 (2012) 212113.

[20] J. Wu, Y. Hirono, X.L. Li, Z.M.M. Wang, J. Lee, M. Benamara, S.Y. Luo, Y.I. Mazur, E.S. Kim, G.J. Salamo, Self-Assembly of Multiple Stacked Nanorings by Vertically Correlated Droplet Epitaxy, Advanced Functional Materials, 24 (2014) 530-535.

[21] T. Mano, K. Watanabe, S. Tsukamoto, H. Fujioka, M. Oshima, N. Koguchi, Fabrication of InGaAs quantum dots on GaAs(0 01 1) by droplet epitaxy, Journal of Crystal Growth, 209 (2000) 504-508.

[22] A. Luque, A. Marti, C. Stanley, Understanding intermediate-band solar cells, Nat Photon, 6 (2012) 146-152.

[23] J. Hong, Z. Hu, M. Probert, K. Li, D. Lv, X. Yang, L. Gu, N. Mao, Q. Feng, L. Xie, J. Zhang, D. Wu, Z. 
Zhang, C. Jin, W. Ji, X. Zhang, J. Yuan, Z. Zhang, Exploring atomic defects in molybdenum disulphide monolayers, Nature Communications, 6 (2015) 6293.

[24] J. Wu, Z.M.M. Wang, V.G. Dorogan, S.B. Li, J. Lee, Y.I. Mazur, E.S. Kim, G.J. Salamo, Effects of rapid thermal annealing on the optical properties of strain-free quantum ring solar cells, Nanoscale Research Letters, 8 (2013) 1-5.

[25] H.F. Lu, L. Fu, G. Jolley, H.H. Tan, S.R. Tatavarti, C. Jagadish, Temperature dependence of dark current properties of InGaAs/GaAs quantum dot solar cells, Applied Physics Letters, 98 (2011) 183509.

[26] W. Katsuyuki, K. Nobuyuki, G. Yoshihiko, Fabrication of GaAs Quantum Dots by Modified Droplet Epitaxy, Japanese Journal of Applied Physics, 39 (2000) L79.

[27] S.M. Hubbard, C.D. Cress, C.G. Bailey, R.P. Raffaelle, S.G. Bailey, D.M. Wilt, Effect of strain compensation on quantum dot enhanced GaAs solar cells, Applied Physics Letters, 92 (2008) 123512.

[28] A. Martí, N. López, E. Antolín, E. Cánovas, A. Luque, C.R. Stanley, C.D. Farmer, P. Díaz, Emitter degradation in quantum dot intermediate band solar cells, Applied Physics Letters, 90 (2007) 233510.

[29] J. Wu, Z.M. Wang, Droplet epitaxy for advanced optoelectronic materials and devices, J Phys D, 47 (2014). 